\title{
¿Qué contenidos sobre alimentación abordan los libros de texto de Ciencias de la Naturaleza en Educación Primaria?
}

\author{
Cristina Gil González y Ángel Luis Cortés Gracia \\ Facultad de Educación. Departamento de Didácticas Específicas, Área de Didáctica \\ de las Ciencias Experimentales. Universidad de Zaragoza. Zaragoza. España
}

[Recibido el 13 de mayo de 2020, aceptado el 18 de julio de 2020]

En este trabajo se han analizado los contenidos sobre alimentación que aparecen en los libros de texto de Educación Primaria y cómo se distribuyen a lo largo de la etapa educativa. El estudio de cinco colecciones de editoriales completas (30 libros de texto de Ciencias de la Naturaleza) ha permitido identificar las diferencias existentes entre las prescripciones curriculares (LOMCE) para la etapa, los tópicos considerados importantes desde una perspectiva teórica y los contenidos que finalmente incluyen los libros de texto analizados. La mayor cantidad y variedad de contenidos se concentra en los tres primeros cursos de Educación Primaria, mientras que en los tres restantes (especialmente 5 ㅇ y $6^{\circ}$ ) se reducen significativamente y algunas editoriales ni siquiera incluyen contenidos sobre alimentación en sus libros.

Palabras clave: alimentación; análisis de contenido; libros de texto; Educación Primaria.

\section{What content on food do the Primary Education textbooks of Natural Sciences address?}

In this work we have analyzed the contents about food that appear in the textbooks of Primary Education and how these contents are distributed throughout the educational stage. The study of five complete editorial collections ( 30 textbooks of Natural Sciences) has allowed us to identify the differences between the curricular prescriptions (LOMCE) for the stage, the topics considered important from a theoretical perspective and the contents that finally include the analyzed textbooks. The greatest amount and variety of content is concentrated in the first three Primary Education courses, while the remaining three (especially 5th and 6th) are significantly reduced and some publishers do not even include content on food in their books.

Keywords: food; content analysis; textbooks; Primary Education.

Para citar el artículo. Gil González, C. y Cortés Gracia, A.L. (2020). ¿Qué contenidos sobre alimentación abordan los libros de texto de Ciencias de la Naturaleza en Educación Primaria? Ápice. Revista de Educación Científica, 4 (2), 17-33. DOI: https://doi.org/10.17979/arec.2020.4.2.6529

Contacto.crisgilgon@unizar.es, acortes@unizar.es 


\section{Introducción}

La alimentación, uno de los procesos esenciales de los seres vivos, es un factor determinante en diferentes aspectos de la vida diaria de las personas. Nuestros hábitos, relaciones sociales y economía individual o familiar son algunos de ellos, siendo especialmente importante en el ámbito de la salud (Cabello et al., 2016, Merelles et al., 2005).

Una visión general sobre los países desarrollados, entre los cuales se encuentra España, permite detectar varios problemas en el ámbito de la alimentación (Del Carmen, 2000). Por un lado, existe una mala educación alimentaria, no por la falta de medios para proporcionar comida, sino de conocimientos de lo que se debe comer (Ejeda, 2009). Ello está trayendo consigo la aparición de casos de malnutrición, carencias nutricionales, desórdenes alimenticios y un aumento en los índices de obesidad, especialmente en la población infantil (Camarero, 2019). En la actualidad, en la mayoría de la población infantil en edad escolar, es evidente el desarrollo de una serie de malos hábitos alimenticios en los que predomina la ingesta de alimentos altamente procesados que está dando lugar a un preocupante incremento en la obesidad y el sobrepeso en nuestro país (entre un $35 \%$ y un $40 \%$ según los últimos estudios), siendo uno de los mayores de Europa (Alba-Martín, 2016; Burgos, 2007; Camarero, 2019).

En España, a pesar de las numerosas investigaciones, proyectos escolares y sanitarios y programas como ALADINO, PERSEO o NAOS, entre otros, que se han elaborado tanto a nivel nacional como autonómico sobre alimentación y nutrición, la obesidad infantil y el sobrepeso prevalecen (Fúster et al., 2009). Los medios de comunicación son uno de los factores que más está influyendo en agravar esta situación (González, 2013; Goris, 2009). La presión publicitaria que ejercen los medios de comunicación para que se consuman determinados alimentos está afectando especialmente a los más pequeños por ser un grupo de edad acrítico y fácil de manipular. Así, modifican sus preferencias, peticiones de compra y hábitos alimentarios, al ser incapaces de entender la intención de la publicidad, y aceptan las afirmaciones publicitarias como hechos (Bosqued et al., 2017; Cairns et al., 2013; Galbraith-Emami y Lobstein, 2013; Royo y Rodríguez, 2015). Ante esta situación, parece necesario tratar la alimentación como un tema de vital importancia en la educación, debiendo abordarlo desde las primeras etapas educativas.

Una buena educación alimentaria logra que el alumnado adopte unos hábitos alimentarios correctos y siga las pautas de una alimentación saludable por lo que, si nos centramos en la etapa de Educación Primaria (en adelante EP), debería realizarse con un nivel de profundidad creciente (Rivadulla-López et al., 2016). Se trata de abordar el tema desde una perspectiva integral que permita combinar conocimientos, actitudes y conductas saludables que promuevan en los niños y niñas un estilo de vida saludable y logre que adquieran una capacidad crítica para elegir una alimentación sana (Vega et al., 2012). Para ello, los materiales curriculares empleados desde antaño por el profesorado para desempeñar su labor docente se han convertido en mediadores clave del proceso de enseñanza y aprendizaje.

A pesar de la diversidad de materiales existentes, como recursos audiovisuales, ordenadores con acceso a Internet, software educativo, unidades didácticas y paquetes con actividades experimentales entre otros, el libro de texto sigue siendo el material educativo más utilizado por el profesorado en muchas aulas y por el alumnado en el hogar (LópezValentín y Guerra-Ramos, 2012; Martínez y Rodríguez, 2010; Rodríguez et al., 2018). En un estudio reciente (Molina y Alfaro, 2019), la mayoría de los docentes de EP indicaban que una de las ventajas del libro de texto es que "supone una guía o esquema claro" para ellos (p. 190). En el área de Ciencias de la Naturaleza, como principales instrumentos pedagó- 
gicos, los libros de texto ejercen una notable influencia en el aprendizaje del alumnado al orientar y dirigir muchas de sus actividades. Además, el profesorado no sólo los emplea como guía, sino que en bastantes ocasiones se convierten en el referente exclusivo del saber científico (Campanario y Otero, 2000; Perales y Jiménez, 2002). Hacen falta análisis sistemáticos sobre el contenido (información textual, imágenes, actividades, ejercicios y otros elementos) así como sobre la naturaleza de sus propuestas pedagógicas y el tratamiento de las competencias para contrarrestar la percepción de los libros de texto que posee buena parte del profesorado (así como padres, madres y estudiantes) como fuentes incuestionables del conocimiento científico y de propuestas didácticas (López-Valentín y Guerra-Ramos, 2012).

De este modo, el objetivo general de este estudio ha consistido en identificar los contenidos sobre alimentación que aparecen en los libros de texto de Ciencias Naturales en Educación Primaria y la distribución de esos contenidos a lo largo de esta etapa.

\section{Marco teórico}

Dada la importancia que tienen la alimentación y la nutrición tanto a nivel educativo como en la vida cotidiana, en muchos países se han puesto en marcha estudios relacionados con las competencias (food competences, Food Standard Agency, 2007), el desarrollo de habilidades (food skills, Region of Waterloo Public Health, 2015, Vanderkooy, 2010) o la alfabetización en alimentación (food literacy, Cullerton et al., 2012; Vidgen y Gallegos 2011). En España, de acuerdo con el enfoque competencial del currículo en las últimas reformas educativas (LOE, LOMCE...), algunos autores han tratado de establecer un marco teórico de referencia para caracterizar las competencias en salud, en general, (proyecto COMSAL, Gavidia, 2016; Pérez de Eulate et al., 2015) y la competencia en alimentación, en particular, (Cabello et al., 2018; España et al., 2014) y, de esta forma, definir el conocimiento que toda persona debería tener al finalizar su educación obligatoria.

Los aspectos científicos y didácticos relacionados con la alimentación y la nutrición han sido objeto de numerosos trabajos desde el punto de vista educativo, en general, y desde la perspectiva de la Didáctica de las Ciencias Experimentales y la Educación para la Salud, en particular (Banet, 2001; Cabello et al., 2009; Cabello et al., 2016; España et al., 2014; García-Barros, 2016; García-Barros y Martínez-Losada, 2009; Garzón et al., 2018; Pellicer, 2019, entre otros). Muchos de los estudios anteriormente citados y buena parte de las propuestas didácticas sobre alimentación y nutrición no abordan la temática de forma integral, sino que se centran en temas concretos de las etapas de Educación Infantil y Primaria (Cubero et al., 2013, 2017; Núñez y Banet, 2000; Thió de Pol, 2011), de Educación Secundaria Obligatoria (Bizzio et al., 2015) e incluso en la formación del profesorado (García-Barros et al., 2010; Rodrigo et al., 2012).

En el sistema educativo español, el bloque 2 del currículo de EP (LOMCE) establece que entre los contenidos mínimos de la asignatura troncal Ciencias de la Naturaleza se deben incluir, entre otros, la función de nutrición, los hábitos saludables y los avances de la ciencia que mejoran la vida. Al mismo tiempo, como estándares de aprendizaje evaluables se incluye la identificación, localización y funcionamiento de los órganos y aparatos relacionados con la nutrición, el conocimiento de las dietas equilibradas, los avances en la producción y conservación de alimentos, entre muchos otros. De este modo, esos contenidos y estándares determinan las competencias que el alumnado debe adquirir en esa etapa educativa alrededor del tema El ser humano y la salud.

Los libros de texto concretan los significados de los contenidos incluidos en el currículo oficial (Gimeno, 2005; Gómez, 2016; Martínez, 2002) como parte de un proceso que trata 
de facilitar la enseñanza y aprendizaje de esos contenidos mediante adaptaciones basadas en criterios pedagógicos y didácticos. Es tal la importancia que tienen los libros de texto en nuestro sistema educativo que, en palabras de algunos autores, "este recurso ha sido algo más que un material de aprendizaje: se ha confundido con el currículum oficial, ha servido para clarificar cambios que se exigían y no se entendían, ha permitido actualizar científicamente al profesorado, ha dado seguridad a los docentes..." (Rodríguez et al., 2018, p. 3102-2). Por este motivo, entre otros, el análisis de los libros de texto ha constituido una línea de trabajo muy importante dentro de la investigación educativa (Aguilera y Perales, 2018; López-Noguero, 2002; Occelli y Valeiras 2013).

Pese a la importancia que se otorga a la alimentación y nutrición tanto en el currículo como en la investigación e innovación educativa, son escasos los trabajos que analizan qué contenidos concretos aparecen y cómo se abordan los mismos en los libros de texto de la educación obligatoria, aunque existen algunos trabajos de referencia en el ámbito de la Educación para la Salud, en general, o bajo el enfoque del análisis de contenidos competenciales (Garzón et al., 2018; Gavidia, 2003; García-Barros y Martínez-Losada, 2005; Torres-García et al., 2018). De este modo, parece interesante plantear una investigación que estudie a nivel descriptivo qué contenidos sobre alimentación y nutrición incluyen los libros de texto de EP, cómo los tratan y cómo se distribuyen esos contenidos a lo largo de toda la etapa.

\section{Metodología}

Para determinar qué contenidos sobre alimentación y nutrición están presentes en los libros de texto de EP se ha procedido a realizar un análisis de contenido (Bardin, 1986; Espín, 2002; López-Noguero, 2002) a partir de una muestra de libros de texto de Ciencias de la Naturaleza de varias de las editoriales más vendidas en España, entendiendo que pueden ser una muestra representativa de los libros de texto españoles (Tabla 1). Se han analizado cinco colecciones completas del periodo 2014-2018 (30 libros, ver Anexo I), centrando el estudio en las unidades temáticas donde deberían aparecer estos contenidos de acuerdo con el currículo oficial, especialmente en el Bloque 2, El ser humano y la salud, aunque se ha revisado el contenido completo de los libros. Evidentemente, también pueden existir contenidos sobre alimentación y nutrición en otras asignaturas troncales y específicas, como Ciencias Sociales o Educación Física, que no han sido objeto de este análisis.

Tabla 1. Nomenclatura y colores utilizados para cada una de las editoriales analizadas

\begin{tabular}{|c|c|c|}
\hline NOMENCLATURA Y COLOR & EDITORIAL \\
\hline Editorial 1 & & Anaya \\
\hline Editorial 2 & & Edebé \\
\hline Editorial 3 & & Edelvives \\
\hline Editorial 4 & & Santillana \\
\hline Editorial 5 & & Vicens Vives \\
\hline
\end{tabular}

Según diversos autores, el análisis de contenido "sólo puede entenderse de una forma completa si lo situamos en el contexto de un diseño de investigación" (López-Noguero, 2002, p. 173). Así, la definición de las unidades de análisis y categorías es uno de sus 
principales problemas, por lo que no existen plantillas de análisis generalizables, ya que dependen de los objetivos de la investigación (Occelli y Valeiras, 2013). Para ello, en cada análisis es necesario inventar o adaptar una técnica adecuada para los intereses de la investigación (Bardin, 1986; Boronat, 2005; Espín, 2002; Izquierdo et al., 2008; Jiménez y Perales, 2001; Laya y Martínez-Losada, 2019; Torres-García et al., 2018).

Antes de realizar este análisis se ha elaborado un sistema inicial de categorías y subcategorías para los temas sobre alimentación que pueden aparecer en los libros de texto (Tabla 2). Para ello, se ha realizado, por un lado, una búsqueda en el currículum de EP (LOMCE y desarrollo autonómico) de los contenidos relacionados con la alimentación que aparecen señalados explícitamente en cada uno de los cursos de la etapa y, por otro, una amplia revisión bibliográfica dirigida a establecer las categorías que tienen importancia desde el punto de vista teórico (Cabello et al., 2016; Del Carmen, 2000; España et al., 2014; Gavidia, 2003; Pérez de Eulate et al., 2015, entre otros). De esta forma, todas esas categorías de interés se han incluido en una plantilla de análisis de elaboración propia que se ha aplicado a la muestra completa de libros de texto. Tras revisar desde una perspectiva cualitativa las colecciones completas de cada una de las editoriales se han encontrado contenidos que no encajaban en las categorías iniciales, por lo que antes de diseñar la plantilla definitiva de análisis se han incorporado varias categorías emergentes (contenidos concretos de esos libros) que no habían sido previstas inicialmente (Tabla 2).

Una vez revisadas las colecciones editoriales a partir del sistema inicial de categorías, añadiendo las emergentes tras la revisión inicial de las colecciones editoriales, se ha llevado a cabo un rediseño del instrumento de análisis. De esta forma, se ha establecido un sistema final de categorías y subcategorías, prestando especial atención a la afinidad existente entre ellas e incluso a su posible ausencia en todas las editoriales. Así pues, varias de las categorías se han englobado dentro de una misma como, por ejemplo, los contenidos conceptuales generales sobre alimentación y nutrición (conceptos de alimentación, nutrición o grupos y tipos de alimentos), el etiquetado nutricional (fecha de caducidad, información nutricional) o las formas de representación de las necesidades nutricionales (rueda, pirámide, tablas). Por otro lado, otras no se han identificado en la revisión inicial, como es el caso de las técnicas de cocinado, consideradas importantes desde el punto de vista teórico pero sin contenidos en los libros analizados (Tabla 2). Para algunos contenidos que aparecían inicialmente en la categorías Otros, como el agua, se ha creado una categoría propia, ya que además de constituir contenidos fundamentales del tema analizado, su notoriedad trasciende al mismo y existen referencias a la importancia del agua para la vida, para la salud, etc. en otros contextos. De este modo, el sistema de categorías y subcategorías con las que finalmente se ha realizado el análisis de contenido aparecen reflejados en la Tabla 3 (ver resultados).

Tras el análisis de los contenidos sobre alimentación que aparecen en cada uno de los cursos en las editoriales señaladas, en la tabla 2 destaca, en primer lugar, la clara diferencia existente entre el tipo y cantidad de contenidos que se consideran importantes desde el punto de vista teórico (un total de 25 categorías) y los que aparecen en el currículum de EP (un total de 13 categorías). Se han identificado ocho categorías emergentes relacionados con algún tipo de contenidos importantes desde el punto de vista teórico y/o curricular. De estas categorías emergentes ninguna presenta afinidad temática con las categorías Etiquetado nutricional, Higiene y hábitos saludables y Otros en las que se han agrupado contenidos durante la primera fase de rediseño de categorías. 
Tabla 2. Elaboración del sistema de categorías de análisis

\begin{tabular}{|c|c|c|c|c|c|}
\hline $\begin{array}{l}\text { Categorías } \\
\text { iniciales }\end{array}$ & $\begin{array}{l}\text { Subcategorías } \\
\text { iniciales }\end{array}$ & $\begin{array}{l}\text { Importantes } \\
\text { desde la } \\
\text { perspectiva } \\
\text { teórica } \\
\end{array}$ & $\begin{array}{l}\text { Presentes en } \\
\text { el currículum }\end{array}$ & $\begin{array}{l}\text { Emergentes } \\
\text { durante el } \\
\text { análisis }\end{array}$ & $\begin{array}{l}\text { Revisadas o } \\
\text { refundidas* }\end{array}$ \\
\hline \multirow{8}{*}{$\begin{array}{c}\text { Conceptos } \\
\text { generales sobre } \\
\text { alimentación y } \\
\text { nutrición }\end{array}$} & $\begin{array}{l}\text { Importancia de } \\
\text { la alimentación }\end{array}$ & $x$ & & & \\
\hline & $\begin{array}{c}\text { Alimentación } \\
\text { vs } \\
\text { nutrición }\end{array}$ & $x$ & $\mathrm{X}$ & & \\
\hline & $\begin{array}{l}\text { Función de } \\
\text { nutrición }\end{array}$ & $x$ & $x$ & & \\
\hline & $\begin{array}{c}\text { Origen de } \\
\text { los alimentos }\end{array}$ & $x$ & & & \\
\hline & $\begin{array}{l}\text { Tipos de } \\
\text { alimentos }\end{array}$ & & & $x$ & \\
\hline & $\begin{array}{l}\text { Aportaciones } \\
\text { nutricionales }\end{array}$ & $\mathrm{X}$ & & & \\
\hline & $\begin{array}{l}\text { Funciones de } \\
\text { los alimentos }\end{array}$ & $x$ & & & \\
\hline & $\begin{array}{l}\text { Grupos de } \\
\text { alimentos }\end{array}$ & $x$ & & & \\
\hline \multirow{3}{*}{$\begin{array}{c}\text { Representación } \\
\text { de las } \\
\text { necesidades } \\
\text { nutricionales }\end{array}$} & $\begin{array}{l}\text { Rueda de los } \\
\text { alimentos }\end{array}$ & $x$ & $x$ & & \\
\hline & $\begin{array}{l}\text { Pirámide de } \\
\text { los alimentos }\end{array}$ & & & & \\
\hline & $\begin{array}{c}\text { Tabla de } \\
\text { composición } \\
\text { de los } \\
\text { alimentos } \\
\end{array}$ & & & $\mathrm{X}$ & \\
\hline \multirow{5}{*}{ Dieta y menú } & $\begin{array}{c}\text { Dieta } \\
\text { saludable y } \\
\text { equilibrada }\end{array}$ & $x$ & $x$ & & $A$ \\
\hline & $\begin{array}{c}\text { Dieta } \\
\text { mediterránea }\end{array}$ & & $x$ & & $A$ \\
\hline & $\begin{array}{l}\text { Tipos de } \\
\text { dietas }\end{array}$ & & & $x$ & A \\
\hline & $\begin{array}{c}\text { Menú } \\
\text { saludable }\end{array}$ & $x$ & $x$ & & $A$ \\
\hline & Raciones & $x$ & & & A \\
\hline \multirow{2}{*}{$\begin{array}{l}\text { Enfermedades, } \\
\text { alergias e } \\
\text { intolerancias } \\
\text { alimentarias }\end{array}$} & $\begin{array}{l}\text { Enfermedades } \\
\text { relacionadas } \\
\text { con la } \\
\text { alimentación }\end{array}$ & $x$ & $x$ & & B \\
\hline & $\begin{array}{c}\text { Alergias e } \\
\text { intolerancias } \\
\text { alimentarias }\end{array}$ & & & $x$ & B \\
\hline
\end{tabular}


Tabla 2. Elaboración del sistema de categorías de análisis. Continuación

\begin{tabular}{|c|c|c|c|c|c|}
\hline $\begin{array}{l}\text { Categorías } \\
\text { iniciales }\end{array}$ & $\begin{array}{c}\text { Subcategorías } \\
\text { iniciales }\end{array}$ & $\begin{array}{l}\text { Importantes } \\
\text { desde la } \\
\text { perspectiva } \\
\text { teórica }\end{array}$ & $\begin{array}{l}\text { Presentes en } \\
\text { el currículum }\end{array}$ & $\begin{array}{c}\text { Emergentes } \\
\text { durante el } \\
\text { análisis }\end{array}$ & $\begin{array}{l}\text { Revisadas o } \\
\text { refundidas* }\end{array}$ \\
\hline \multirow{8}{*}{$\begin{array}{c}\text { Elaboración, } \\
\text { cocinado y } \\
\text { consumo }\end{array}$} & $\begin{array}{l}\text { Ingredientes } \\
\text { de elaboración }\end{array}$ & & & $\mathrm{X}$ & C \\
\hline & $\begin{array}{l}\text { Técnicas de } \\
\text { procesado de } \\
\text { alimentos }\end{array}$ & & & $x$ & C \\
\hline & $\begin{array}{c}\text { Aditivos } \\
\text { alimentarios }\end{array}$ & $x$ & & & C \\
\hline & Conservantes & & & $\mathrm{X}$ & C \\
\hline & $\begin{array}{l}\text { Métodos de } \\
\text { conservación }\end{array}$ & $x$ & $x$ & & C \\
\hline & $\begin{array}{l}\text { Envasado de } \\
\text { alimentos }\end{array}$ & $x$ & & & C \\
\hline & $\begin{array}{l}\text { Técnicas de } \\
\text { cocinado** }\end{array}$ & $x$ & & & $C^{* *}$ \\
\hline & $\begin{array}{l}\text { Formas de } \\
\text { consumo }\end{array}$ & & & $x$ & C \\
\hline \multirow{2}{*}{$\begin{array}{l}\text { Etiquetado } \\
\text { nutricional }\end{array}$} & $\begin{array}{l}\text { Fecha de } \\
\text { caducidad }\end{array}$ & $x$ & & & $\mathrm{D}$ \\
\hline & $\begin{array}{c}\text { Información } \\
\text { nutricional }\end{array}$ & $x$ & $x$ & & D \\
\hline \multirow{3}{*}{$\begin{array}{c}\text { Higiene } \\
\text { y hábitos } \\
\text { saludables }\end{array}$} & $\begin{array}{c}\text { Hábitos } \\
\text { saludables }\end{array}$ & $x$ & $x$ & & $E$ \\
\hline & $\begin{array}{l}\text { Seguridad } \\
\text { e higiene } \\
\text { alimentaria }\end{array}$ & $x$ & $x$ & & $E$ \\
\hline & $\begin{array}{c}\text { Ocio, descanso } \\
\text { y actividad } \\
\text { física }\end{array}$ & $\mathrm{X}$ & $x$ & & $E$ \\
\hline \multirow{4}{*}{ Otros } & Agua & & $x$ & & $\mathrm{~F}$ \\
\hline & $\begin{array}{l}\text { La } \\
\text { alimentación } \\
\text { en el mundo }\end{array}$ & $x$ & & & G \\
\hline & $\begin{array}{c}\text { Gastronomía } \\
\text { de otras } \\
\text { culturas }\end{array}$ & $x$ & & & G \\
\hline & $\begin{array}{l}\text { Publicidad } \\
\text { alimentaria }\end{array}$ & $x$ & & & $\mathrm{H}$ \\
\hline
\end{tabular}

* Categorías revisadas y refundidas para el análisis final: A. Dieta y menú; B. Enfermedades, alergias e intolerancias alimentarias; C. Elaboración, cocinado y consumo; D. Etiquetado nutricional. E; Higiene y hábitos saludables; F. Agua; G. La cultura y su relación con la alimentación; H. Publicidad alimentaria.

** Categoría ausente en todas las editoriales. 


\section{Resultados}

Una vez aplicada la plantilla de análisis con el sistema final de categorías, se ha realizado una aproximación cuantitativa a la frecuencia de aparición de los contenidos en el conjunto de libros analizados ( $\mathrm{N}=30$ ). Como se puede observar en la Tabla 3, a nivel general, destacan algunas categorías que aparecen en el currículo de EP y que son importantes desde el punto de vista teórico, como Hábitos saludables, Seguridad e Higiene alimentaria $(n=27)$, Dieta saludable y equilibrada $(n=25)$ y La nutrición humana $(n=23)$, por ser las tres que poseen contenidos con más frecuencia de aparición en todas las editoriales. Esto quiere decir que existen contenidos de esas categorías de forma explícita en la mayor parte de los libros analizados, siendo los correspondientes a la higiene y hábitos saludables los más citados (27/30). De manera contraria, la Tabla de composición de los alimentos tan solo aparece citada en un libro y la categoría Publicidad alimentaria, importante desde el punto de vista teórico, tan solo tiene contenidos explícitos en dos libros de diferentes editoriales de 40 y 60 curso, respectivamente.

Tabla 3. Categorías y subcategorías finales y frecuencia de aparición de contenidos. Se ha considerado la existencia de cualquier tipo de contenidos de la categoría en cada libro analizado, hasta un máximo de 30 (6 libros de 5 editoriales).

\begin{tabular}{|c|c|c|}
\hline Categorías finales & Subcategorías finales & $\begin{array}{l}\text { Frecuencias de } \\
\text { aparición }(\mathrm{N}=30)\end{array}$ \\
\hline \multirow{6}{*}{$\begin{array}{l}\text { Conceptos generales sobre } \\
\text { alimentación y nutrición }\end{array}$} & Importancia de la alimentación & 19 \\
\hline & La nutrición humana & 23 \\
\hline & Origen de los alimentos & 9 \\
\hline & Tipos de alimentos & 7 \\
\hline & Funciones y aportaciones nutricionales & 19 \\
\hline & Grupos de alimentos & 21 \\
\hline \multirow{3}{*}{$\begin{array}{l}\text { Representación de las } \\
\text { necesidades nutricionales }\end{array}$} & Rueda de los alimentos & 14 \\
\hline & Pirámide de los alimentos & 14 \\
\hline & Tabla de composición de los alimentos & 1 \\
\hline Dieta y salud & Dieta saludable y equilibrada & 25 \\
\hline $\begin{array}{l}\text { Enfermedades, alergias e } \\
\text { intolerancias alimentarias }\end{array}$ & $\begin{array}{c}\text { Enfermedades, alergias e intolerancias } \\
\text { alimentarias }\end{array}$ & 19 \\
\hline Elaboración, cocinado y consumo & Elaboración, cocinado y consumo & 15 \\
\hline Etiquetado nutricional & Etiquetado nutricional & 17 \\
\hline Higiene y hábitos saludables & $\begin{array}{l}\text { Hábitos saludables, seguridad e } \\
\text { higiene alimentaria }\end{array}$ & 27 \\
\hline Agua & Agua & 16 \\
\hline Publicidad alimentaria & Publicidad alimentaria & 2 \\
\hline $\begin{array}{c}\text { La cultura y su relación con la } \\
\text { alimentación }\end{array}$ & $\begin{array}{c}\text { La cultura y su relación con la } \\
\text { alimentación }\end{array}$ & 7 \\
\hline
\end{tabular}

En cuanto al número de categorías distintas identificadas en los contenidos presentes en los libros de cada curso y editorial (figura 1), destacan los cursos de 2ㅇ, 3으 y 4 을 de EP por ser los que más variedad de contenidos sobre alimentación contienen, especialmente 30 EP. Las editoriales 2 y 4 son las que más contenidos de diferentes categorías muestran en cada uno de ellos con un total de 13 categorías, mientras que la editorial 1 es la que menos diversidad de contenidos muestra en estos tres cursos. 


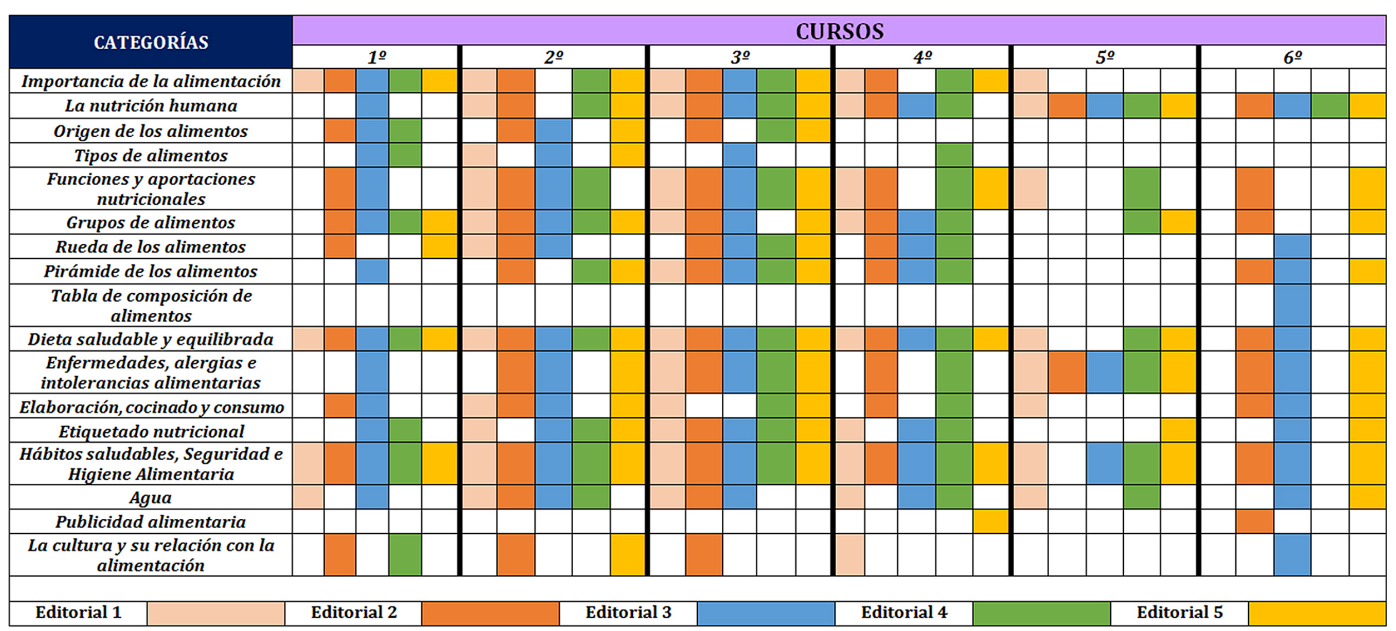

Figura 1. Distribución de las categorías (subcategorías) de contenidos sobre alimentación por editoriales y cursos a lo largo de la etapa de EP.

En 10 EP, a pesar de la escasa diversidad de contenidos mostrados por las editoriales 1 y 5 en particular, los contenidos sobre alimentación que muestran todas las editoriales son muy similares a los que muestran en los siguientes tres cursos de la etapa. En los dos últimos cursos se observa un claro descenso en la cantidad y variedad de contenidos en general, especialmente en 50 EP, destacando la ausencia efectiva de contenidos en el libro de 60 EP de la editorial 1, aunque esta editorial es la que más contenidos de categorías distintas presenta en 50 (8/17), frente a las editoriales 2 y 3 , con contenidos casi anecdóticos.

Así, de forma general (figura 1), en el conjunto de editoriales se observa un aumento progresivo en el número de categorías tratadas a lo largo de los tres primeros cursos (39 en 10,57 en 2 ㅇ y 59 en 3 o). Sin embargo, en los tres cursos restantes el número de categorías que incluyen cada una de las editoriales se ve claramente reducido e incluso desaparecen (42 en $4 \div$, 26 en 5 ㅇ y 32 en 6ㅇ).

Si se observa cada uno de los cursos, se puede destacar que en 1ㅇ EP, de los contenidos reflejados en el currículo para este nivel, los correspondientes a las categorías Dieta saludable y equilibrada y Hábitos saludables y Seguridad e Higiene Alimentaria, son los únicos mostrados por todas las editoriales, junto a la Importancia de la alimentación, como categoría genérica señalada en los referentes teóricos. Sin embargo, categorías también consideradas importantes desde el punto de vista teórico, como Enfermedades, alergias e intolerancias alimentarias y Pirámide de los alimentos, tan sólo son mostradas por la editorial 3 formando parte de las actividades de los temas correspondientes.

En $2 \circ$ curso, las categorías presentes en el currículum de EP Dieta saludable y equilibrada y Hábitos saludables, Seguridad e Higiene Alimentaria y La nutrición humana son reflejadas por todas las editoriales excepto por la editorial 3, que no trata explícitamente la nutrición. En el caso de los contenidos que son considerados importantes desde el punto de vista teórico, la categoría Publicidad alimentaria no aparece en ninguna editorial y la categoría La cultura y su relación con la alimentación tan solo es mostrada por las editoriales 2 y 5 de forma muy resumida.

En 3 ㅡ, curso académico en el que más contenidos diferentes sobre alimentación muestran las editoriales analizadas, la categoría Publicidad alimentaria tampoco es tratada por ninguno de los libros en sus contenidos, y La cultura y su relación con la alimentación solo es mostrada por la editorial 2 de forma muy reducida. Sin embargo, todas las categorías reflejadas en el currículo en este nivel son mostradas por todas las editoriales. Además, 
las cinco editoriales muestran contenidos de categorías curriculares no exigidas explícitamente para este nivel como Funciones y aportaciones nutricionales y Etiquetado nutricional.

En lo que respecta a 4 으, al igual que ocurre en 20 y 3 으, las únicas categorías del currículo exigidas en este curso y representadas en todas las editoriales son Dieta saludable y equilibrada y Hábitos saludables, Seguridad e Higiene Alimentaria. Sin embargo, otras como La nutrición humana, Rueda de los alimentos, Enfermedades, alergias e intolerancias alimentarias y Etiquetado nutricional, que además son importantes desde el punto de vista teórico, no tienen contenidos en varios de los libros. Los contenidos sobre La cultura y su relación con la alimentación y Publicidad alimentaria, a pesar de su importancia desde la perspectiva teórica son reflejados nada más que por las editoriales 1 y 5 respectivamente.

Finalmente, en 50 y 60 EP se evidencia un marcado descenso en el número de contenidos de las diferentes categorías que tratan cada una de las editoriales en sus libros. La categoría Dieta saludable y equilibrada, a pesar de ser mostrada en el resto de cursos por todas las editoriales y formar parte de las categorías exigidas en el currículo para estos niveles, solo aparece de manera muy puntual en las actividades planteadas en la unidad didáctica y como título de uno de sus apartados, con escasos contenidos. Igualmente, las categorías La nutrición humana y Enfermedades, alergias e intolerancias alimentarias señaladas en el currículo para 50 y 60 EP son tratadas por todas las editoriales en 50 y de forma desigual en 6‥ La categoría Etiquetado nutricional solo aparece en las editoriales 3 y 5 de forma muy puntual como información adicional al resto de los contenidos.

Por último, hay que señalar que algunos contenidos que tienen importancia desde el punto de vista teórico (España et al., 2014 y Gavidia, 2003, entre otros), en 5o y 60 EP, y, especialmente en 50 , apenas son tratados por ninguna editorial. Éstos aparecen solamente en uno de estos dos cursos y de una manera casi anecdótica, como es el caso de las categorías Rueda de los alimentos y La cultura y su relación con la alimentación, las cuales tan solo son mostradas por la editorial 3 en 6 o EP como parte de información extra. Lo mismo ocurre con la categoría Publicidad alimentaria, que aparece únicamente mencionada en la portada de la unidad didáctica sobre alimentación en la editorial 2 en 6 EP. La categoría Grupos de alimentos aparece dentro de apartados en los que se explican otros conceptos como los nutrientes. Finalmente, los contenidos de la categoría Agua, que muestran 4 de las 5 editoriales analizadas en 50 o en 60 EP, aparece en relación a los nutrientes aportados por los alimentos y en la adquisición de hábitos saludables.

\section{Discusión y consideraciones finales}

El análisis realizado ha permitido establecer hasta 17 categorías/subcategorías diferentes en relación con los contenidos de alimentación que aparecen en las cinco colecciones completas de los libros de texto de Ciencias de la Naturaleza de EP del periodo 2014-2018.

A nivel general, todos los contenidos prescritos en el currículo de EP son abordados a lo largo de la etapa en alguno de los cursos, aunque no necesariamente por todas las editoriales ni en los cursos que indican las órdenes autonómicas que detallan su distribución temporal durante la etapa. Este es el caso, por ejemplo, de la categoría Rueda de los alimentos que aparece mencionada explícitamente en 50 EP y, sin embargo, las editoriales que la muestran lo hacen principalmente en 30 y 4 으. EP.

En relación al marco teórico de referencia (España et al., 2014; Gavidia, 2003, Pérez de Eulate et al., 2015, entre otros), las categorías Rueda de los alimentos y Pirámide de los alimentos no aparecen en todos los cursos. Aquellos en los que se muestran lo hacen de forma desigual y no en todas las editoriales. A esta situación debemos unir el debate 
existente sobre el uso de esas representaciones (pirámide tradicional y rueda de los alimentos) y el mensaje confuso que puede llegar a interpretar la ciudadanía. Para ello se han propuesto alternativas como la nueva pirámide de la alimentación saludable de la Sociedad Española de Nutrición Comunitaria (Aranceta et al., 2016) o el modelo tridimensional que propone la Fundación Iberomericana de Nutrición (Gil et al., 2015). Otra propuesta es el reciente modelo del plato para comer saludable de Harvard (https://www. hsph.harvard.edu/nutritionsource/healthy-eating-plate/). A pesar de que los nutricionistas consideran más acertados estos enfoques que la pirámide o rueda tradicionales, estos modelos apenas tienen representación en los libros de texto. La categoría Publicidad alimentaria, tan solo aparece reflejada en 40 y 60 EP por una editorial en cada curso. Tal y como se ha comentado en la introducción de este trabajo, la publicidad tiene una gran influencia en los hábitos de consumo y la toma de decisiones de las niñas y niños, por lo que consideramos especialmente grave su ausencia en los contenidos de los libros de texto que tienen como referencia.

Al margen de los motivos concretos que cada editorial posea para decidir incluir o no determinados contenidos en sus libros de texto, los resultados de este análisis parecen indicar que éstos no siempre responden a las prescripciones curriculares o a una profunda revisión teórica sobre su adecuación. Por ejemplo, en 3 EP aparecen tratados los contenidos de la mayor parte de las categorías en todas las editoriales. Sin embargo, el currículo no especifica el abordaje de todos esos contenidos en ese curso concreto. Por ello, parece más bien una coincidencia (acordada o no) entre editoriales más que una decisión basada en criterios curriculares. De la misma forma, en 5 o EP los contenidos son más escasos en los libros de texto a pesar de la existencia de los mismos en el currículo para ese curso.

En cuanto a la distribución de los contenidos a lo largo de la etapa existe una importante reducción en la variedad de contenidos que muestran cada una de las editoriales conforme avanzan los cursos académicos. De esta forma, en los primeros cursos, y, especialmente en 3 o EP, es donde más variedad de contenidos aparecen. Por el contrario, los cursos de 50 y 60 EP (especialmente 5) son los que menos variedad de contenidos reflejan. Como se ha comentado anteriormente, resulta significativa la escasa existencia de contenidos en 50 y 60 EP siendo que en el currículum de EP existen contenidos específicos para estos cursos, como Etiquetado nutricional y Rueda de los alimentos, entre otros, y que algunos de los trabajos de referencia (Burgos, 2007; Cabello et al., 2016, 2018; Del Carmen, 2010; Gavidia, 2003) hacen especial énfasis en la importancia de desarrollar la competencia en alimentación especialmente en el periodo de 10 a 17 años.

Teniendo en cuenta que nuestro sistema educativo apuesta por el respeto y reconocimiento de la pluralidad cultural y de la interculturalidad como elemento enriquecedor de la sociedad, llama la atención la casi inexistente presencia de La cultura y su relación con la alimentación en los temas relacionados con la alimentación. Por otro lado, nuestra sociedad es cada vez más sensible a los problemas que generan algunos alimentos y/o sus ingredientes (enfermedades, obesidad, alergias, intolerancias, etc.) que tienen que ver con la composición de éstos, y por tanto relacionados con los ingredientes, información nutricional y etiquetado, o con los métodos de conservación de los mismos. Por ello, parece necesario que se trabajen de forma progresiva hasta los últimos cursos de Educación Primaria estos aspectos, integrando cuestiones sociales y de interacción con el medio y con la salud, tanto de forma individual como colectiva (Rivadulla-López et al., 2016), de manera que se pueda desarrollar la competencia en alimentación a la que aluden autores como Cabello et al. (2016). La escasez en los últimos cursos de esta etapa de todos estos contenidos, al menos en los libros de texto de Ciencias de la Naturaleza, es difícilmente justificable desde una perspectiva teórica, pedagógica o curricular. 
En conclusión, es evidente la importancia que tienen los conocimientos sobre alimentación y nutrición tanto a nivel educativo como en la vida cotidiana. Dado el relevante papel que para muchos profesores tienen los libros de texto en el proceso de enseñanza y aprendizaje, resulta necesaria la revisión del contenido de los mismos de manera que se garantice una Educación Alimentaria efectiva que reduzca o, al menos, ralentice los problemas alimentarios existentes en la sociedad actual y permita desarrollar el pensamiento crítico frente a la influencia ejercida por los medios de comunicación en al ámbito de la salud y alimentación.

\section{Agradecimientos}

A los revisores, por sus acertados comentarios y sugerencias. Grupo de referencia BEAGLE Investigación en Didáctica de Ciencias Naturales (Gobierno de Aragón y Fondo Social Europeo) Instituto de Investigación en Ciencias Ambientales de Aragón (IUCA/UNIZAR). Proyecto EDU2016-76743-P (MINECO). Cristina Gil González disfruta de un contrato predoctoral del Gobierno de Aragón (ORDEN IIU/1408/2018), Programa Operativo FSE Aragón 2014-2020, Construyendo Europa desde Aragón.

\section{Referencias bibliográficas}

Alba-Martín, R. (2016). Prevalencia de obesidad infantil y hábitos alimentarios en educación primaria. Revista electrónica trimestral de enfermería, 42, 40-51. Recuperado de http://scielo.isciii.es/pdf/eg/v15n42/clinica3.pdf

Aguilera, D. y Perales, J. (2018). El libro de texto, las ilustraciones y la actitud hacia la Ciencia del alumnado: percepciones, experiencias y opiniones del profesorado. Enseñanza de las ciencias, 36(3), 41-58. DOI: https://doi.org/10.5565/rev/ensciencias.2423

Aranceta, J., Arija, V., Maíz, E., Martínez de Victoria, E., Ortega, R. M., Pérez-Rodrigo, C., Quiles, J., Rodríguez, A., Román, B., Salvador, G., Tur, J. A., Varela, G. y Serra, L. (2016). Guías alimentarias para la población española (SENC, diciembre 2016); la nueva pirámide de la alimentación saludable. Nutrición Hospitalaria, 33(8), 1-48 DOI: http://dx.doi.org/10.20960/nh.827

Banet, E. (2001). Los procesos de nutrición humana. Madrid: Síntesis Educación.

Bardin, L. (1986). El análisis de contenido. Madrid: Akal.

Bizzio, M. A., Pereira, R. A., Nuñez, G. I. y Morales, L. M. (2015). Aprendiendo a seleccionar alimentos en el recreo escolar. Alambique Didáctica de las Ciencias Experimentales, 79, 73-78.

Boronat Mundina, J. (2005). Análisis de contenido. Posibilidades de aplicación en la investigación educativa. Revista Interuniversitaria de Formación del Profesorado, 19(2), 157-174.

Bosqued, J. M., López, L., Moyá, A. y Royo, M. A. (2016). La publicidad alimentaria dirigida a menores en España. Alimentación, nutrición y salud, 23(1), 19-25.

Burgos N. (2007). Alimentación y nutrición en edad escolar. Revista Digital Universitaria $8(4), 2-7$.

Cabello, A., Blanco, A. y España, E. (2009). Una hipótesis de trabajo para investigar el progreso en la comprensión de la alimentación humana por parte de los alumnos. Enseñanza de las Ciencias, Número extra VIII Congreso, 1729-1735. 
Cabello, A., España, E. y Blanco, A. (2016). La competencia en alimentación. Barcelona: Octaedro.

Cabello, A., España, E. y Blanco, A. (2018). Developing a Human Nutrition Learning Progression. International Journal of Science and Mathematics Education, 16, 1269-1289. DOI: https://doi.org/10.1007/s10763-017-9838-y

Cairns, G., Angus, K., Hastings, G. y Caraher, M. (2013). Systematic reviews of the evidence on the nature, extent and effects of food marketing to children. A retrospective summary. Appetite, 62, 209-215. DOI: https://doi.org/10.1016/j.appet.2012.04.017

Camarero Gómez, A. (2019). Alimentación y educación saludable. Presentación. Cuadernos de Pedagogía, 497, 6-8.

Campanario, J. M. y Otero, J. (2000). La comprensión de los libros de texto de ciencias. En J. Perales y P. Cañal (Eds.), Didáctica de las Ciencias Experimentales. Teoría y práctica de la enseñanza de las Ciencias (pp. 323-338). Alcoy: Ed. Marfil.

Cubero, J., Franco-Reynolds, L., Calderón, M. A., Caro, B., Rodrigo, M. y Ruiz, C. (2017). El desayuno escolar; una intervención educativa en alimentación y nutrición saludable. Didáctica de las Ciencias Experimentales y Sociales, 32(1), 171-182. DOI: https://doi.org/10.7203/DCES.32.4546

Cubero, J., Guerra, M., Costillo, E., Pozo, E. y Ruiz, C. (2013). Análisis del desayuno en una población de escolares del 3o ciclo de Primaria; un recurso didáctico en Educación para la Salud. Campo abierto: Revista de educación, 32(2), 145-153.

Cullerton, K., Vidgen, H. y Gallegos, D. (2012). A review of food literacy interventions targeting disadvantaged young people. Recuperado de http://eprints.qut.edu. au/53753/1/food_literacy_interventions_review_final.pdf

Del Carmen, L. (2000). La alimentación: algo más que ingerir alimentos. Aula de innovación educativa, 92, 6-8.

Ejeda, J. M. (2009). El conocimiento sobre alimentación en la formación inicial de los maestros. Tesis doctoral. Universidad Complutense de Madrid. Recuperado de https://eprints.ucm.es/8375/1/T30669.pdf

España, E., Cabello, A. y Blanco, A. (2014). La competencia en alimentación. Un marco de referencia para la educación obligatoria. Enseñanza de las Ciencias, 32(3), 611-629. DOI: https://doi.org/10.5565/rev/ensciencias.1080

Espín, J. V. (2002). El análisis de contenido: una técnica para explorar y sistematizar información. XXI, Revista de Educación, 4, 95-105. Recuperado de http://hdl.handle. net/10272/1913

Food Standards Agency (2007). Food Competency framework: food skills and knowledge for children and young people by age of 7-9, 11-12, 14 and 16+. Recuperado de http://www.food.gov.uk/multimedia/pdfs/competencyria.pdf

Fúster, F., Ribes, M. A., Bardón, R. y Marino E. (2009). Análisis cuantitativo de las noticias de alimentación en la prensa madrileña en 2006. Revista española de documentación científica, 32(1), 99-115. DOI: https://doi.org/10.3989/redc.2009.1.664

Galbraith-Emami, S. y Lobstein, T. (2013). The impact of initiatives to limit the advertising of food and beverage products to children: a systematic review. Obesity Reviews, 14, 960-974. DOI: https://doi.org/10.1111/obr.12060 
García-Barros, S. (2016). La nutrición: una función imprescindible para mantener la vida. Marco reflexivo didáctico. Alambique Didáctica de las Ciencias Experimentales, 84, 7-12.

García-Barros, S. y Martínez-Losada, C. (2005). La nutrición en textos escolares del último ciclo de primaria y primero de secundaria. Enseñanza de las Ciencias, Extra 2005. Recuperado de https://ddd.uab.cat/pub/edlc/edlc_a2005nEXTRA/ edlc_a2005nEXTRAp234nuttex.pdf

García-Barros, S. y Martínez-Losada, M. C. (2009). La nutrición: una función vital que invita a la reflexión didáctica. Aula de Innovación Educativa, 183-184, 37-40.

García-Barros, S., Martínez-Losada, C. y Rivadulla, J. C. (2010). La percepción medioambiental del profesorado de primaria en el tema de la nutrición humana. Revista Eureka sobre enseñanza y divulgación de la ciencia, 7, 286-296. DOI: http://dx.doi. org/10.25267/Rev_Eureka_ensen_divulg_cienc.2010.v7.iextra.11

Garzón, A., Talavera, M. y Gavidia, V. (2018). Niveles de competencia en alimentación y actividad física en los libros de texto de Educación Secundaria Obligatoria. Didáctica de las Ciencias Experimentales y Sociales, 36, 61-78. DOI: https://doi.org/10.7203/ DCES.36.12186

Gavidia, V. (2003). La educación para la salud en los manuales escolares españoles. Revista Española de Salud Pública, 77, 275-285.

Gavidia, V. (2016). La Salut en el curriculum educatiu. Viure en Salut, 107, 8-9.

Gil, A., Ruiz-López, M. D., Fernández-González, M., Martínez de Victoria, E. (2015).Guía FINUT de estilos de vida saludable: más allá de la Pirámide de los Alimentos. Nutrición Hospitalaria, 31(5), 2313-2323. DOI: https://doi.org/10.3305/nh.2015.31.5.8803

Gimeno Sacristán, J. (2005). El currículum: ¿ Los contenidos de la enseñanza o un análisis de la práctica? En J. Gimeno Sacristán y A. I. Pérez Gómez (eds.), Comprender y transformar la enseñanza (pp. 137-170). Madrid: Morata.

Gómez Mendoza, M. A. (2016). El libro de texto escolar: espacios, lectura, hábitos digitales y recepción. Profesorado. Revista de currículum y formación de profesorado, 20(1), 33-47.

González, C. (2013). Publicidad de alimentos y mensajes de salud: un estudio exploratorio. Ámbitos. Revista Internacional de Comunicación, 23. Recuperado de http://hdl. handle.net/10045/34303

Goris, J. M. (2009). Television food advertising and the prevalence of childhood overweight and obesity: a multicountry comparison. Public Health Nutrition, 13(7), 1003-1012.

Izquierdo, M., Márquez, C. y Gouvea, G. (2008). A proposal for textbooks analysis: rethorical structures. Science Education International, 19(2), 209-218.

Jiménez Valladares, J. D. y Perales Palacios, F. J. (2001). Aplicación del análisis secuencial al estudio del texto escrito e ilustraciones de los libros de física y química de la ESO. Enseñanza de las Ciencias, 19(1), 3-19.

Laya Iglesias, P. y Martínez-Losada, C. (2019) La competencia científica en los libros de texto de Educación Primaria. Ápice. Revista de Educación Científica, 3(1), 71-83. DOI: https://doi.org/10.17979/arec.2019.3.1.5000

López-Noguero, F. (2002). El análisis de contenido como método de investigación. XXI, Revista de Educación, 4, 167-179. Recuperado de http://hdl.handle.net/10272/1912 
López-Valentín, D. M. y Guerra-Ramos, M. T. (2012). Análisis de las actividades de aprendizaje incluidas en libros de texto de ciencias naturales para Educación Primaria utilizadas en México. Enseñanza de las Ciencias, 31(2), 173-191. DOI: https://doi. org/10.5565/rev/ec/v31n2.815

Martínez Bonafé, J. (2002). Políticas del libro escolar. Madrid: Ediciones Morata.

Martínez Bonafé, J. y Rodríguez Rodríguez, J. (2010). El curriculum y el libro de texto escolar. Una dialéctica siempre abierta. En J. Gimeno Sacristán (comp.), Saberes e incertidumbres sobre el currículum (pp. 246-268). Madrid: Morata.

Merelles, T., Costa, A., Sánchez, A. y Ruano, L. (2005). La educación nutricional desde la Atención Primaria. En C. Vázquez, C. López-Nomdedeu y A. de Cos, Alimentación y nutrición: manual teórico-práctico (pp. 273-283). Madrid: Díaz de Santos.

Molina Puche, S. y Alfaro Romero, A. (2019). Ventajas e inconvenientes del uso del libro de texto en las aulas de Educación Primaria. Percepciones y experiencias de docentes de la Región de Murcia. Revista Electrónica Interuniversitaria de Formación del Profesorado, 22(2), 179-197. DOI: http://dx.doi.org/10.6018/reifop.22.2.332021

Núñez, F. y Banet, E. (2000). Aprender sobre la alimentación para desarrollar hábitos y actitudes saludables en el alumnado de primaria. Aula de Innovación Educativa, 92, 9-14.

Occelli, M. y Valeiras, N. (2013). Los libros de texto de ciencias como objeto de investigación: una revisión bibliográfica. Enseñanza de las Ciencias, 31(2), 133-152. DOI: https://doi.org/10.5565/rev/ec/v31n2.761

Pellicer, C. (2019). La salud integral del alumno, una asignatura pendiente en su curriculum vital. Cuadernos de pedagogía, 497, 3-5.

Perales, F. J. y Jiménez, J. D. (2002). Las ilustraciones en la enseñanza-aprendizaje de las ciencias. Análisis de libro de texto. Enseñanza de las Ciencias, 20(3), 369-386.

Pérez de Eulate, L., Llorente, E., Gavidia, V., Caurín, C. y Martínez, M. J. (2015). ¿Qué enseñar en la educación obligatoria acerca de la alimentación y la actividad física? Un estudio con expertos. Enseñanza de las Ciencias, 33(1), 85-100. DOI: https://doi. org/10.5565/rev/ensciencias.1355

Region of Waterloo Public Health (2015). Food skills of Waterloo Region. Changes over 6 years. Recuperado de https://www.regionofwaterloo.ca/en/health-and-wellness/ resources/Documents/FoodSkills_WR.pdf

Rivadulla-López, J. C., García-Barros, S. y Martínez-Losada, C. (2016). Los mapas conceptuales como instrumento para analizar las ideas de los estudiantes de Maestro de Educación Primaria sobre qué enseñar de nutrición humana en Educación Primaria. Revista complutense de educación, 27(3), 1247-1269. DOI: https://doi.org/10.5209/ rev_RCED.2016.v27.n3.47704

Rodrigo, M., Ejeda, J. M. y Caballero, M. (2012). Una década enseñando e investigando en Educación Alimentaria para Maestros. Revista Complutense en Educación, 24(2), 243-265. DOI: https://doi.org/10.5209/rev_rced.2013.v24.n2.42078

Rodríguez Moreno, J., Pro Bueno, A. y Molina Jaén, M. D. (2018). Opinión de los docentes sobre el tratamiento de las competencias en los libros de texto de Ciencias de la Naturaleza en Educación Primaria. Revista Eureka sobre Enseñanza y Divulgación de las Ciencias, 18(3), 3102. DOI: https://doi.org/10.25267/Rev_Eureka_ensen_ divulg_cienc.2018.v15.i3.3102 
Royo, M. A. y Rodríguez, F. (2015). Publicidad alimentaria y salud. Estado de la situación en España. Mediterráneo Económico, 27, 319-330.

Thió de Pol, C. (2011). El día a día: La comida más allá de la nutrición: autonomía, autoestima, responsabilidad. Aula de infantil, 59, 44-45.

Torres-García, M., Marrero-Montelongo, M., Navarro-Rodríguez, C. y Gavidia, V. (2018). ¿Cómo abordan los textos de Educación Primaria la competencia en alimentación y actividad física? Revista Eureka sobre Enseñanza y Divulgación de las Ciencias, 15(1), 1103. DOI: https://doi.org/10.25267/Rev_Eureka_ensen_divulg_cienc.2018. v15.i1.1103

Vanderkooy, P. (2010). Food skills of Waterloo Region adults. Fireside Chat Presentation. Recuperado de http://www.chnet-works.ca/index.php?option=com_phocadownlo ad\&view=category\&id=7\%3Afireside-chat-presentations-2010\&ltemid=13\&lang=e n\&limitstart $=20>$

Vega, M. R., Ejeda, J. M. y Caballero, M. (2012). Una década enseñando e investigando en Educación Alimentaria para Maestros. Revista Complutense de Educación, 24(2), 243-265.

Vidgen, H. y Gallegos, D. (2011). What is food literacy and does it influence what we eat: a study of Australian food experts. Queensland University of Technology. Recuperado de http://eprints.qut.edu.au/45902/> 
ANEXO 1. Listado de libros analizados.

\begin{tabular}{|c|c|c|}
\hline Editorial & Curso & ISBN \\
\hline \multirow{6}{*}{ ANAYA } & 10 & $978-84-698-3868-6$ \\
\hline & 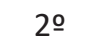 & $978-84-698-3875-4$ \\
\hline & 3음 & $978-84-698-4224-9$ \\
\hline & $4 \div$ & $978-84-698-0754-5$ \\
\hline & 50 & $978-84-698-4383-3$ \\
\hline & 6은 & $978-84-698-8090-8$ \\
\hline \multirow{6}{*}{$E D E B E ́$} & 10 & $978-84-683-1409-9$ \\
\hline & 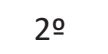 & $978-84-683-2102-8$ \\
\hline & 3응 & $978-84-683-1461-7$ \\
\hline & $4 \div$ & $978-84-683-2042-7$ \\
\hline & 5은 & $978-84-683-1462-4$ \\
\hline & 6은 & $978-84-683-2043-4$ \\
\hline \multirow{6}{*}{ EDELVIVES } & 10 & $978-84-263-9299-2$ \\
\hline & 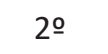 & $978-84-263-9591-7$ \\
\hline & 3응 & $978-84-263-9319-7$ \\
\hline & 4 & $978-84-263-9623-5$ \\
\hline & 50 & $978-84-263-9363-0$ \\
\hline & 6은 & $978-84-263-9653-2$ \\
\hline \multirow{6}{*}{ SANTILLANA } & 10 & $978-84-680-1163-9$ \\
\hline & 20 & $978-84-680-1485-2$ \\
\hline & 3응 & $978-84-680-4736-2$ \\
\hline & 40 & $978-84-680-2919-1$ \\
\hline & 50 & $978-84-680-2554-4$ \\
\hline & 6은 & $978-84-680-2920-7$ \\
\hline \multirow{6}{*}{ VICENS VIVES } & 10 & $978-84-682-5474-6$ \\
\hline & 20 & $978-84-682-5487-6$ \\
\hline & 3응 & $978-84-682-2070-3$ \\
\hline & $4 ㅇ$ & $978-84-682-2840-2$ \\
\hline & 50 & $978-84-682-1504-4$ \\
\hline & 60 & $978-84-682-1558-7$ \\
\hline
\end{tabular}


